\title{
Symmetry energy properties of neutron-rich nuclei from the coherent density fluctuation model applied to nuclear matter calculations with Bonn potentials
}

\author{
I. C. Danchev, ${ }^{1}$ A. N. Antonov, ${ }^{2}$ D. N. Kadrev, ${ }^{2}$ M. K. Gaidarov, ${ }^{2}$ P. Sarriguren,${ }^{3}$ and E. Moya de Guerra ${ }^{4}$ \\ ${ }^{1}$ Department of Physical and Mathematical Sciences, \\ School of Arts and Sciences, University of Mount Olive, \\ 652 R.B. Butler Dr., Mount Olive, NC 28365, USA \\ ${ }^{2}$ Institute for Nuclear Research and Nuclear Energy, \\ Bulgarian Academy of Sciences, Sofia 1784, Bulgaria \\ ${ }^{3}$ Instituto de Estructura de la Materia, IEM-CSIC, Serrano 123, E-28006 Madrid, Spain \\ ${ }^{4}$ Departamento de Estructura de la Materia, Física Térmica y Electrónica, and IPARCOS, \\ Facultad de Ciencias Físicas, Universidad Complutense de Madrid, Madrid E-28040, Spain
}

\begin{abstract}
We derive the values of nuclear symmetry energy, its components, as well as pressure in finite nuclei at saturation density from their corresponding values in nuclear matter obtained in nonrelativistic Brueckner-Hartree-Fock calculations with the realistic Bonn B and Bonn CD potentials using the coherent density fluctuation model in the framework of a self-consistent Skyrme-HartreeFock plus BCS method. We focus on three isotopic chains of spherical nuclei ( $\mathrm{Ni}, \mathrm{Sn}$, and $\mathrm{Pb})$ and compare our results with those obtained with an effective Brueckner density-dependent potential. The role of the three-body forces on the considered quantities is also studied and discussed.
\end{abstract}

PACS numbers: 21.60.Jz, 21.65.Ef, 21.10.Gv

\section{INTRODUCTION}

The basic properties of many-body quantummechanical systems with strongly interacting particles primarily depend on the equation of state (EOS). In particular, this concerns symmetric and asymmetric nuclear matter including astrophysical objects such as neutron stars and the gravitational wave events caused by binary black hole and binary neutron star mergers (see, e.g., Ref. [1]). A very important theoretical problem is to determine the EOS from underlying nuclear interactions using two-, three-, and many-body nucleon interactions derived in various theoretical approaches. It is necessary in many cases to calculate the EOS to extreme conditions of high density and high neutronproton asymmetry. This inevitably leads to the necessity to have a knowledge of the nuclear symmetry energy $S^{N M}(\rho)$ as one of the most important quantities that determines the structure and the pressure of the nuclear matter (NM). The $S^{N M}(\rho)$ is the difference between the binding energy per particle in pure neutron matter and in symmetric nuclear matter. Many calculations of the EOS and $S^{N M}(\rho)$ have been performed using various realistic models of the nucleon-nucleon interactions with parameters adjusted so as to reproduce the observed nucleon-nucleon $(N N)$ scattering phase shifts. Here we note (see [2]) the variational calculations in Ref. [3] with Argonne $V_{14}$ potentials [4] and the earlier Brueckner-Hartree-Fock (BHF) calculations using one-boson-exchange potential [5, 6] with Reid [7] and Paris potentials 8]. It has been concluded in Ref. 2] that the differences in the predictions of the $S^{N M}(\rho)$ at high densities are not caused by the many-body method used, but rather by the various models used for the $N N$ interactions.

In what follows we concentrate on the non-relativistic many-body BHF calculations in NM with various potentials. It was initiated in the sixties by Brueckner 9, 10] and Bethe [11], and later by Haftel and Tabakin [12] and Erkelenz et al. [13, 14] with the objective to derive the saturation properties of nuclear matter from first principles and obtain the experimentally observed nuclear binding energy. Self-consistent calculations of the density dependence of the nuclear symmetry energy were carried by Brueckner, Coon, and Dabrowski in Ref. [15].

Substantial progress in the study of the equation of state of nuclear matter was achieved in the nineties with relativistic extensions of that approach and employment of one-boson-exchange nucleon-nucleon potentials [16 18. The investigations of $\beta$ stability of nuclear matter [19] at high densities brought to center stage the need of accurate knowledge of the density dependence of the nuclear symmetry energy and many-body calculations in both non-relativistic [2] and relativistic [20] framework were carried out using modern realistic potentials.

A comparative study of different approaches for the properties of asymmetric nuclear matter has been presented in Refs. 21, 22]. These various approximation schemes are shown to lead to rather similar predictions for the energy per nucleon of symmetric and asymmetric nuclear matter at high densities and large proton-neutron asymmetries.

The study of finite nuclei has further significance for the development of nuclear density functionals. Very recently, Shen et al. have developed and realized an application of relativistic BHF theory for finite nuclear systems [23]. They showed that further improvements in building $a b$ initio relativistic energy functionals are needed (inclusion of higher orders of the hole-line expansion, more precise relativistic $N N$ interactions, efforts to develop relativistic chiral $N N$ interactions) to go, for instance, to heavier systems. 
The main task of our work is to employ the modern realistic Bonn $\mathrm{B}$ and Bonn $\mathrm{CD}$ potentials on the basis of the non-relativistic approach used by Engvik et al. [2]. First, we obtain the density dependence of the NM symmetry energy for a range of densities relevant to the present study and then we use the coherent density fluctuation model (CDFM) [24, 25] to extract the symmetry energy and pressure in three isotopic chains of (finite) spherical nuclei with input nuclear densities derived in self-consistent Skyrme-Hartree-Fock + BCS calculations.

In our previous works 26 30 the CDFM allowed us to make the transition from nuclear matter to finite nuclei in the studies of the nuclear symmetry energy (NSE) for spherical [26] and deformed [27] nuclei, as well as for $\mathrm{Mg}$ isotopes [28] using the Brueckner energy-density functional (EDF) of asymmetric nuclear matter 31]. In our work [29] we used a similar method to investigate the temperature dependence of the NSE for isotopic chains of even-even $\mathrm{Ni}, \mathrm{Sn}$, and $\mathrm{Pb}$ nuclei following the local density approximation [32 35] and using instead of the Brueckner EDF, the Skyrme EDF with SkM* and SLy4 forces. In our work [30] the volume and surface contributions to the NSE and their ratio were calculated within the CDFM using two EDF's, namely the Brueckner 31] and Skyrme (see Ref. [36]) ones. Recently our results for the mentioned quantities have been given in Ref. [37].

The structure of this article is the following. In Sec. II we present the common definitions of the symmetry energy and properties of nuclear matter which characterize its density dependence around normal nuclear matter density. A brief description of the nonrelativistic Brueckner-Hartree-Fock formalism for calculating the ground-state properties of symmetric nuclear matter and extracting the density dependence of the nuclear symmetry energy in quadratic approximation of the isospin polarization is given, as well. In Sec. [II] we present a brief account of the formalism of the CDFM which allows us to relate the intrinsic quantities in nuclear matter to their corresponding ones in finite nuclei using different phenomenological potentials. In Sec. IV we present and discuss the results of our calculations of the already mentioned quantities of spherical nuclei for three different isotopic chains of even-even $\mathrm{Ni}(A=74-84)$, Sn $(A=124-152)$, and $\mathrm{Pb}(A=206-214)$ nuclei within the CDFM formalism in the framework of Skyrme HF + BCS obtained from two different nuclear potentials for nuclear matter interactions: on one hand the modern realistic Bonn B and Bonn CD potentials [16, 17] and on the other the time-honored Brueckner's density dependent potential [31, 38]. The effect of the Bonn B and Bonn CD potentials plus the microscopic three-body forces (TBF) on the symmetry energy and related quantities on the example of $\mathrm{Ni}$ isotopes is also estimated and discussed. The conclusions of the present work are given in Sec. V

\section{THE KEY EOS PARAMETERS IN NUCLEAR MATTER AND IN FINITE NUCLEI}

\section{A. Semi-empirical Bethe-Weizsäcker formula for nuclear matter}

The semi-empirical Bethe-Weizsäcker formula [39, 40] captures the essential dependence of the finite nucleus ground state on isospin asymmetry (polarization). This formula may be viewed as a Taylor series expansion in the energy per particle for nuclear matter in terms of the isospin asymmetry $\delta=\left(\rho_{n}-\rho_{p}\right) / \rho$, in which the densitydependent coefficient in front of the quadratic term defines the so-called symmetry energy $S^{N M}(\rho)$

$$
E(\rho, \delta)=E(\rho, 0)+S^{N M}(\rho) \delta^{2}+O\left(\delta^{4}\right)+\cdots,
$$

where $\rho=\rho_{n}+\rho_{p}$ is the baryon density with $\rho_{n}$ and $\rho_{p}$ denoting the neutron and proton densities, respectively (see, e.g., [41, 42]). Odd powers of $\delta$ are forbidden by the isospin symmetry and the terms proportional to $\delta^{4}$ and higher orders are found to be negligible.

Near the saturation density $\rho_{0}$ the energy of isospinsymmetric matter $E(\rho, 0)$ and the symmetry energy, $S^{N M}(\rho)$, can be expanded as

$$
E(\rho, 0)=E_{0}+\frac{K^{N M}}{18 \rho_{0}^{2}}\left(\rho-\rho_{0}\right)^{2}+\cdots
$$

and

$$
\begin{aligned}
S^{N M}(\rho)= & \left.\frac{1}{2} \frac{\partial^{2} E(\rho, \delta)}{\partial \delta^{2}}\right|_{\delta=0}=a_{4}+\frac{p_{0}^{N M}}{\rho_{0}^{2}}\left(\rho-\rho_{0}\right) \\
& +\frac{\Delta K^{N M}}{18 \rho_{0}^{2}}\left(\rho-\rho_{0}\right)^{2}+\cdots .
\end{aligned}
$$

The parameter $a_{4}$ is the symmetry energy at equilibrium $\left(\rho=\rho_{0}\right)$. The pressure $p_{0}^{N M}$

$$
p_{0}^{N M}=\left.\rho_{0}^{2} \frac{\partial S^{N M}}{\partial \rho}\right|_{\rho=\rho_{0}}
$$

and the curvature $\Delta K^{N M}$

$$
\Delta K^{N M}=\left.9 \rho_{0}^{2} \frac{\partial^{2} S^{N M}}{\partial \rho^{2}}\right|_{\rho=\rho_{0}}
$$

of the nuclear symmetry energy at $\rho_{0}$ govern its density dependence and thus provide important information on the properties of the nuclear symmetry energy at both high and low densities.

The Bethe-Weizsäcker semi-empirical mass formula for the nuclear ground-state energy per nucleon describes both properties of (infinite) nuclear matter as well as finite nuclei [43, 44]:

$$
\begin{aligned}
E(A, Z)= & -B+E_{S} A^{-1 / 3}+S(A) \frac{(N-Z)^{2}}{A^{2}}+E_{C} \frac{Z^{2}}{A^{4 / 3}} \\
& +E_{\text {dif }} \frac{Z^{2}}{A^{2}}+E_{e x} \frac{Z^{4 / 3}}{A^{4 / 3}}+a \Delta A^{-3 / 2}
\end{aligned}
$$


where

$$
S(A)=\frac{S^{V}(A)}{1+\frac{S^{S}(A)}{S^{V}(A)} A^{-1 / 3}}=\frac{S^{V}(A)}{1+\frac{A^{-1 / 3}}{\kappa(A)}}
$$

with

$$
\kappa(A) \equiv \frac{S^{V}(A)}{S^{S}(A)} .
$$

In Eq. (6) $B \simeq 16 \mathrm{MeV}$ is the binding energy per particle of bulk symmetric matter at saturation. $E_{S}, E_{C}, E_{d i f}$, and $E_{e x}$ are coefficients that correspond to the surface energy of symmetric matter, the Coulomb energy of a uniformly charged sphere, the diffuseness correction, and the exchange correction to the Coulomb energy, while the last term gives the pairing corrections $(\Delta$ is a constant and $a=+1$ for odd-odd nuclei, 0 for odd-even, and -1 for even-even nuclei). $S^{V}$ is the volume symmetry energy parameter and $S^{S}$ is the modified surface symmetry energy in the droplet model (see Ref. [44], where it is defined by $S^{S *}$ ).

In our previous work 29] we have studied the temperature dependence of the nuclear symmetry energy $S(A, T)$, including its volume and surface [37] contributions as derived from Brueckner [31, 38] and Skyrme [36] energy density functionals. In the present work we extract the symmetry energy coefficients in $\mathrm{Ni}, \mathrm{Sn}$, and $\mathrm{Pb}$ from many-body Brueckner-Hartree-Fock ground-state calculations of nuclear matter with realistic potentials (Bonn $\mathrm{B}$ and Bonn $\mathrm{CD})$ at zero temperature $(T=0 \mathrm{MeV})$ by applying the CDFM using the finite nuclei densities obtained in self-consistent Hartree-Fock + BCS calculations with Skyrme effective interactions (SLy4, Sk3, and SGII).

Here we would like to comment on the following point. In the present work (and also in our previous paper [37]) we use Eq. (7) as a relation between the symmetry energy $S(A)$ and its volume $S^{V}(A)$ and surface $S^{S}(A)$ components. As mentioned above, this relation is given in the droplet model, e.g., in Ref. [44]. However, Eq. (7) is different from the relation in another approach used in e.g., Refs. [45 48], and also in our work [30]. In the latter works the relation between $S(A)$ and its components $S^{V}(A)$ and $S^{S}(A)$ is $S^{V}(A) /\left\{1+\left[S^{V}(A) / S^{S}(A)\right] A^{-1 / 3}\right\}$, which contains a ratio in the second term in the denominator $\left[S^{V}(A) / S^{S}(A)\right] A^{-1 / 3}$, while in Eq. (7) the ratio is $\left[S^{S}(A) / S^{V}(A)\right] A^{-1 / 3}$. In the nuclear matter limit, when $A \rightarrow \infty$ and $S^{S} / S^{V} \rightarrow 0$, the symmetry energy in Eq. (7) has the correct limit $S \rightarrow S^{V}$. In this limit the ratio $\left[S^{V}(A) / S^{S}(A)\right] A^{-1 / 3}$ is not well determined and to get the right nuclear matter limit one has to impose the condition that the surface coefficient $S^{S}(A)$ goes to zero more slowly than $A^{-1 / 3}$ as $A \rightarrow \infty$. To avoid this constraint we adopt in our present work (and also in Ref. [37]) the relation from the droplet model [Eq. (7)].

Also, at large $A$ Eq. (7) can be written in the known form (see Ref. [40]):

$$
S(A) \simeq S^{V}-\frac{S^{S}}{A^{1 / 3}}
$$

From Eqs. (7) and (8) follow the relations of $S^{V}(A)$ and $S^{S}(A)$ with $S(A)$ :

$$
S^{V}(A)=S(A)\left(1+\frac{1}{\kappa(A) A^{1 / 3}}\right)
$$

$$
S^{S}(A)=\frac{S(A)}{\kappa(A)}\left(1+\frac{1}{\kappa(A) A^{1 / 3}}\right) .
$$

Due to the choice of Eq. (7), Eqs. (10) and (11) are different from Eqs. (26) and (27) in Ref. [30]. This leads to different results for $S^{V}(A)$ and $S^{S}(A)$ obtained in 30] and those of the present work which will be shown in Sec. IV. We emphasize, however, that the results for the symmetry energy $S(A)$ in this work, as well as in Refs. [30] and [26], should be the same because they are obtained from identical equations, namely, Eq. (27) in Sec. III of the present work, Eq. (20) in [30], and Eq. (28) in Ref. [26]. As can be expected, the differences concern the values of $S^{V}(A)$ and $S^{S}(A)$.

Furthermore, it has been argued (Refs. [45, 49]) that the ratio of the volume to the surface energy coefficients is given by the following integral of the symmetry energy function of density $S(\rho)$ :

$$
\kappa(A)=\frac{S^{V}(A)}{S^{S}(A)}=\frac{3}{r_{0}} \int d r \frac{\rho(r)}{\rho_{0}}\left\{\frac{S^{N M}\left(\rho_{0}\right)}{S^{N M}[\rho(r)]}-1\right\}
$$

in the local density approximation to the symmetry energy. In Eq. (12) $\rho(r)$ is the half-infinite nuclear matter density, $\rho_{0}$ is the nuclear matter equilibrium density, and $r_{0}$ is the radius of the nuclear volume per nucleon. The latter two quantities are related by

$$
\frac{4 \pi r_{0}^{3}}{3}=\frac{1}{\rho_{0}}
$$

In Section IV are presented our results for the volume and surface components of the nuclear symmetry energy and their ratio obtained in calculations based on relationships from this section and within the CDFM (see Section [II).

\section{B. Ground-state properties of dense nuclear matter calculated with Bonn B and Bonn CD nuclear potentials}

In this subsection we give a short overview of the formalism employed in obtaining the density dependence of the symmetry energy of nuclear matter. Details can be seen in the original works of Engvik et al. 2], and also of Haftel and Tabakin 12], Erkelenz et al. [14], Machleidt [16, 18], and Song et al. [50, 51]. Excellent pedagogical reviews can be found in M. Baldo [52] and the lectures of Hjorth-Jensen [53] at the Twelfth Summer School in Nuclear Physics at the University of California, Santa Cruz in 2000 . 
The symmetry energy in nuclear matter $S^{N M}(\rho)$ as a function of density (neglecting higher than quadratic terms in isospin asymmetry) can be approximated with

$$
S^{N M}(\rho)=\mathcal{E}(\rho, \delta=1)-\mathcal{E}(\rho, \delta=0),
$$

where $\mathcal{E}(\rho, \delta=1)$ is the ground state energy per nucleon of completely isospin polarized (i.e. neutron matter) at density $\rho$ and $\mathcal{E}(\rho, \delta=0)$ is the ground-state energy per nucleon of isospin unpolarized (i.e. isospin symmetric) matter. From Eqs. (4) and (14) it follows the relationship for the pressure $p_{0}^{N M}(\rho)$.

The calculation of the ground-state energies per nucleon at different density and polarization is carried out using the reaction matrix $G$, which is a solution of the Bethe-Goldstone equation for various isospin polarization fractions $\delta$ :

$$
G(\omega, \delta)=V+V \frac{Q(\delta)}{\omega-H_{0}} G(\omega, \delta),
$$

where $\omega$ is the unperturbed energy of the interacting nucleons, $V$ is the free $N N$ potential, $H_{0}$ is the unperturbed energy of the intermediate scattering states, and $Q(\delta)$ is the Pauli operator preventing scattering into occupied states. Only ladder diagrams with intermediate two-particle states are included in Eq. (15).

Having obtained a self-consistent solution of BetheGoldstone equation, one can construct the bound state energies per nucleon at different density and isospin asymmetry (polarization) $\delta$

$$
\mathcal{E}(\rho, \delta)=\mathcal{T}+\mathcal{U}(\rho, \delta)
$$

with the kinetic energy

$$
\mathcal{T}=\frac{3}{10 m k_{F}^{3}}\left(k_{F p}^{5}+k_{F n}^{5}\right),
$$

where $m$ is the effective nucleon mass and $k_{F}$ is the total Fermi momentum. The Fermi momenta $k_{F p}$ and $k_{F n}$ for protons and neutrons, respectively, are related to the total nuclear density $\rho$ and isospin asymmetry (polarization) $\delta$ by

$$
\begin{aligned}
\rho & =\frac{2}{3 \pi^{2}} k_{F}^{3}=\frac{(1+\delta)}{2} \rho+\frac{(1-\delta)}{2} \rho \\
& =\frac{1}{3 \pi^{2}} k_{F n}^{3}+\frac{1}{3 \pi^{2}} k_{F p}^{3} .
\end{aligned}
$$

The contribution of the potential energy $\mathcal{U}$ to the total energy per particle can be written in the form:

$$
\begin{aligned}
\mathcal{U}(\rho, \delta) & =\frac{1}{2 \rho} \frac{1}{(2 \pi)^{6}} \sum_{a, b=(p n)} \int_{0}^{k_{F a}} d^{3} k_{a} \int_{0}^{k_{F b}} d^{3} k_{b} \\
& \times\left\langle k_{a} k_{b}\left|G\left(\omega=\epsilon_{a}\left(k_{a}\right)+\epsilon_{b}\left(k_{b}\right)\right)\right| k_{a} k_{b}\right\rangle,
\end{aligned}
$$

where $\epsilon_{a}$ and $\epsilon_{b}$ are the nucleon single-particle energies. Using this approach we have carried out first nonrelativistic Brueckner-Hartree-Fock calculations of the symmetry energy using Bonn B and Bonn CD potentials from [16, 17]. Our results are summarized in Fig. 1. They are in line with previous studies which confirm the nearly linear dependence of the symmetry energy in nuclear matter with density [2, 20]. In the same figure are presented also the results when two-body Bonn B potential from Ref. 54] and two-body Bonn CD potential from Ref. [55], as well as the results for $S^{N M}(\rho)$ obtained by the Nijmegen II and Argonne $V_{18}$ [56] potentials taken from Ref. 2] are used. We note that in Fig. 11 the TBF are not included. Here we would like to emphasize that when comparing the results in Fig. 1 one has to keep in mind their dependence on the choices of the auxiliary potentials and of the methods of calculations. In the paper of Wang et al. 54 BHF calculations are performed (with Bonn B two-body potential and microscopic TBF) using gap and continuous auxiliary potentials that lead to different results, namely, the symmetry energy under the gap choice is smaller than that under the continuous choice. In Fig. 1 the result of [54] with a gap auxiliary potential is compared with our result using also gap auxiliary potential and Bonn B potential from [16]. We should note the existing small difference between our result and that from Ref. [54]. The reason for this is mainly the difference between the estimations made in our work and those in Ref. [54] of the contribution of the ${ }^{1} P_{1}$ partial wave to the energy as a function of the density. For instance, for the density $\rho=0.17 \mathrm{fm}^{-3}$ our estimation is close to that in Table 9.2 of Ref. [16] but it is about $3 \mathrm{MeV}$ larger than that in Table 1 of Ref. [54]. We should add, however, that the noted difference is within the uncertainty in the experimental value of the symmetry energy (e.g. $S=32 \pm 6 \mathrm{MeV}$ given in [55]). The calculations of Soma and Bozek [55] are performed not within the BHF method but using the self-consistent inmedium T-matrix approach implemented with Bonn CD and Nijmegen potentials plus the three-nucleon Urbana interaction. It is found in Ref. [55] that the results of their approach for the symmetry energy in the case of the Bonn CD potential is $S=30-32 \mathrm{MeV}$. In Fig. 2 are given the results of our calculations for the pressure in nuclear matter based on Eqs. (4) and (14) for both Bonn potentials.

In the end of this section we would like to comment on the role of the three-body forces. It is well known that the standard BHF formalism involving only twobody forces does not reproduce correctly the empirical saturation point of nuclear matter [57]. The studies of the TBF effects on the properties of symmetric NM and pure neutron matter in Refs. [54, 55, 58] were performed for Argonne $V_{18}$, CD Bonn, and Bonn B two-body potentials, which are among the few most accurate NN interactions. The latter two potentials are object of the present work and, in principle, of primary interest to investigate the dependence of the nuclear EOS on the twoand three-body forces and their impact on the characteristics of NM for finite nuclei. Indeed, the saturation density and the energy per particle of nuclear matter can be 
improved by including the TBF to $\left(0.17 \mathrm{fm}^{-3}\right.$ and -15.9 $\mathrm{MeV})$ 54] and to $\left(0.185 \mathrm{fm}^{-3}\right.$ and $\left.-15.5 \mathrm{MeV}\right)$ [58] for the Bonn B and Bonn CD potentials, respectively. For comparison, the corresponding saturation points in the case of Brueckner EDF are $\left(0.204 \mathrm{fm}^{-3}\right.$ and $\left.-16.57 \mathrm{MeV}\right)$. In Fig. 3 are compared the results for the symmetry energy as a function of the density from the calculations within the BHF method for Bonn B potential [54] (with a gap choice) and within the in-medium T-matrix approach for Bonn CD potential [55] with and without TBF. In general, as it can be seen from Fig. 3. the TBF play an important role in determining the high-density behavior of the symmetry energy and its effect leads to a strong stiffening of the symmetry energy at high densities. A confirmation of this fact can be also found in Ref. [59], where the TBF play a key role in determining the coefficient $b$ [see Eq. (3) and Table I of Ref. 59]], which is responsible for the high-density behavior of the EOS and whose values vary significantly when two-body or threebody forces are accounted for in quantum Monte Carlo calculations of the neutron star mass-radius relationship.

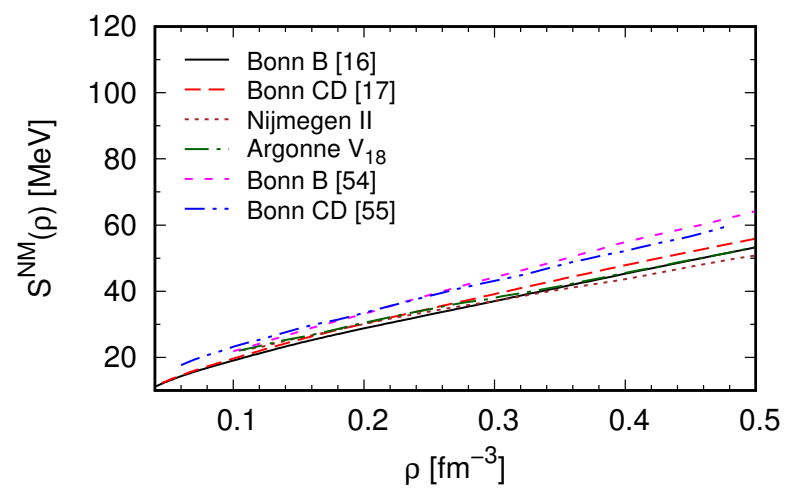

FIG. 1: Density dependence of the symmetry energy calculated with Bonn B and Bonn CD potentials from [16, 17], as well as from [54, 55] and with Nijmegen II and Argonne $V_{18}$ potentials from [2]. We note that all the curves correspond to two-body forces.

\section{NUCLEAR EOS PARAMETERS OF FINITE NUCLEI IN THE CDFM}

In what follows we calculate the key EOS parameters in finite nuclei, i.e. the pressure $p_{0}$ at saturation density $\rho_{0}$ and the nuclear symmetry energy and its surface and volume components (see Refs. [24, 25, 30]) using the CDFM. The latter is a natural extension of the Fermigas model and is based on the $\delta$-function approximation of the generator coordinate method [60]. The model includes nucleon-nucleon correlations of collective type. In general, it allows us to make the transition from quantities in nuclear matter to the corresponding ones in finite nuclei. In the present work it is applied to our studies of the symmetry energy and its components. In the CDFM

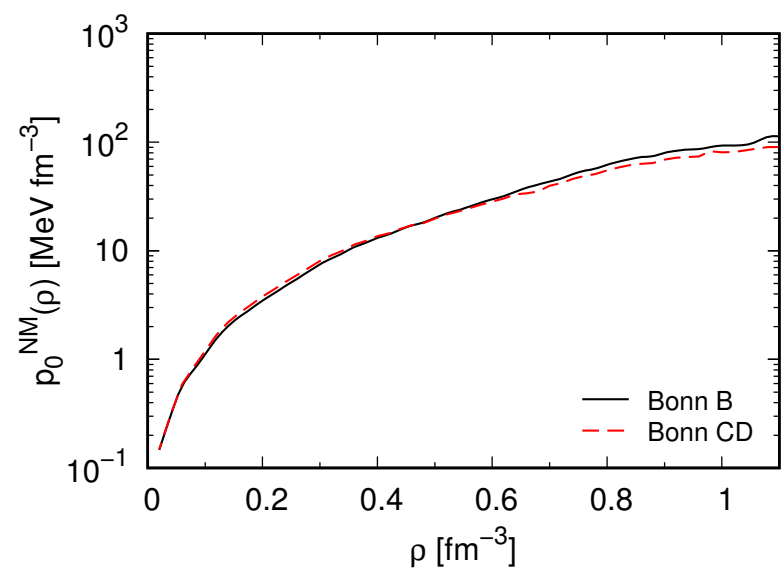

FIG. 2: Density dependence of the pressure in nuclear matter calculated with Bonn B and Bonn CD potentials from [16, 17].

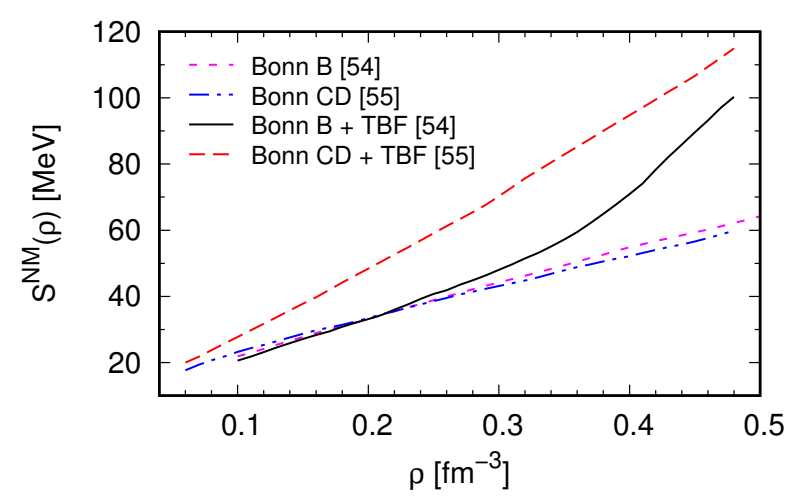

FIG. 3: Density dependence of the symmetry energy calculated with Bonn B [54] and Bonn CD [55] potentials with twoand three-body forces (TBF).

the one-body density matrix $\rho\left(\mathbf{r}, \mathbf{r}^{\prime}\right)$ is a coherent superposition of the one-body density matrices $\rho_{x}^{N M}\left(\mathbf{r}, \mathbf{r}^{\prime}\right)$ for spherical "pieces" of nuclear matter ("fluctons") with densities $\rho_{x}(\mathbf{r})=\rho_{0}(x) \Theta(x-|\mathbf{r}|)$ and $\rho_{0}(x)=3 A / 4 \pi x^{3}$. It has the form:

$$
\rho\left(\mathbf{r}, \mathbf{r}^{\prime}\right)=\int_{0}^{\infty} d x|\mathcal{F}(x)|^{2} \rho_{x}^{N M}\left(\mathbf{r}, \mathbf{r}^{\prime}\right)
$$

with

$$
\begin{aligned}
\rho_{x}^{N M}\left(\mathbf{r}, \mathbf{r}^{\prime}\right) & =3 \rho_{0}(x) \frac{j_{1}\left(k_{F}(x)\left|\mathbf{r}-\mathbf{r}^{\prime}\right|\right)}{\left(k_{F}(x)\left|\mathbf{r}-\mathbf{r}^{\prime}\right|\right)} \\
& \times \Theta\left(x-\frac{\left|\mathbf{r}+\mathbf{r}^{\prime}\right|}{2}\right) .
\end{aligned}
$$

In (21) $j_{1}$ is the first-order spherical Bessel function and

$$
k_{F}(x)=\left(\frac{3 \pi^{2}}{2} \rho_{0}(x)\right)^{1 / 3} \equiv \frac{\beta}{x}
$$

with

$$
\beta=\left(\frac{9 \pi A}{8}\right)^{1 / 3} \simeq 1.52 A^{1 / 3}
$$


is the Fermi momentum of the nucleons in the flucton with a radius $x$. The density distribution in the CDFM has the form:

$$
\rho(\mathbf{r})=\int_{0}^{\infty} d x|\mathcal{F}(x)|^{2} \rho_{0}(x) \Theta(x-|\mathbf{r}|) .
$$

It follows from (24) that in the case of monotonically decreasing local density $(d \rho / d r \leq 0)$ the weight function $|\mathcal{F}(x)|^{2}$ can be obtained from a known density (theoretically or experimentally obtained):

$$
|\mathcal{F}(x)|^{2}=-\left.\frac{1}{\rho_{0}(x)} \frac{d \rho(r)}{d r}\right|_{r=x}
$$

with normalization

$$
\int_{0}^{\infty} d x|\mathcal{F}(x)|^{2}=1
$$

We have shown in our previous works [26, 27, 30] by applying the CDFM that both nuclear symmetry energy and pressure in finite nuclei can be obtained from infinite nuclear matter at temperature $T=0 \mathrm{MeV}$ by weighting it with $|\mathcal{F}(x)|^{2}$ :

$$
\begin{aligned}
& S(A)=\int_{0}^{\infty} d x|\mathcal{F}(x)|^{2} S^{N M}[\rho(x)], \\
& p_{0}(A)=\int_{0}^{\infty} d x|\mathcal{F}(x)|^{2} p_{0}^{N M}[\rho(x)] .
\end{aligned}
$$

Self-consistency requires that when this procedure is applied to quantities referring to (infinite) nuclear matter, the weight function reduces to Dirac delta function. For example, when the condition for self-consistency is applied to the density $\rho(|\mathbf{r}|)$ and the symmetry energy $S^{N M}[\rho(|\mathbf{r}|)]$ in nuclear matter it leads from Eq. (24) and Eq. (27) to the trivial identities:

$$
\begin{aligned}
\rho^{N M}(|\mathbf{r}|, x) & =\int_{0}^{\infty} d x^{\prime} \delta\left(x^{\prime}-x\right) \rho_{0}\left(x^{\prime}\right) \Theta\left(x^{\prime}-|\mathbf{r}|\right) \\
& =\rho_{0}(x) \Theta(x-|\mathbf{r}|) . \\
S^{N M}\left[\rho^{N M}(|\mathbf{r}|, x)\right] & =\int_{0}^{\infty} d x^{\prime} \delta\left(x^{\prime}-x\right) S^{N M}\left[\rho^{N M}\left(|\mathbf{r}|, x^{\prime}\right)\right] \\
& =S^{N M}\left[\rho_{0}(x) \Theta(x-|\mathbf{r}|)\right],
\end{aligned}
$$

In Refs. [26, 27, 30] we used expressions for $S^{N M}[\rho(x)]$ and $p_{0}^{N M}[\rho(x)]$ derived for nuclear matter from an effective density-dependent Brueckner potential [31, 38]:

$$
\begin{aligned}
S^{N M}(x)= & 41.7 \rho_{0}^{2 / 3}(x)+b_{4} \rho_{0}(x) \\
& +b_{5} \rho_{0}^{4 / 3}(x)+b_{6} \rho_{0}^{5 / 3}(x), \\
p_{0}^{N M}(x)= & 27.8 \rho_{0}^{5 / 3}(x)+b_{4} \rho_{0}^{2}(x) \\
& +\frac{4}{3} b_{5} \rho_{0}^{7 / 3}(x)+\frac{5}{3} b_{6} \rho_{0}^{8 / 3}(x),
\end{aligned}
$$

where

$$
b_{4}=148.26, \quad b_{5}=372.84, \quad b_{6}=-769.57 .
$$

In the present work using the CDFM we take nuclear matter values of the considered parameters and use them to deduce the corresponding values in finite nuclei. We apply the CDFM in the framework of a self-consistent Skyrme-Hartree-Fock plus BCS method to calculate the volume and surface contributions to the symmetry energy and their ratio, as well as the pressure, in spherical nuclei of the $\mathrm{Ni}, \mathrm{Sn}$, and $\mathrm{Pb}$ isotopic chains. In our approach to calculate $\kappa(A)$, i.e the ratio $S^{V}(A) / S^{S}(A)$ (see also Ref. [30]) we start from Danielewicz's formula Eq. (12). In it we make an approximation replacing the density $\rho(r)$ for the half-infinite nuclear matter in the integrand by the density distribution of a finite nucleus. We replace the latter by the expression in the CDFM [Eq. (24)]. Concerning the term $S^{N M}[\rho(r)]$, when use is made of Eq. (29) and Eq. (30) the formula for $\kappa(A)$ takes the form

$$
\begin{aligned}
\kappa(A)= & \frac{3}{r_{0} \rho_{0}} \int_{0}^{\infty} d x|\mathcal{F}(x)|^{2} \rho_{0}(x) \\
& \times \int_{0}^{x} d r\left\{\frac{S^{N M}\left(\rho_{0}\right)}{S^{N M}\left[\rho_{0}(x)\right]}-1\right\},
\end{aligned}
$$

which leads to

$$
\kappa(A)=\frac{3}{r_{0} \rho_{0}} \int_{0}^{\infty} d x|\mathcal{F}(x)|^{2} x \rho_{0}(x)\left\{\frac{S^{N M}\left(\rho_{0}\right)}{S^{N M}\left[\rho_{0}(x)\right]}-1\right\},
$$

where the right-hand side of Eq. (35) is an onedimensional integral over $x$. The latter is the radius of the "flucton" that is perpendicular to the nuclear surface.

The weight function $|\mathcal{F}(x)|^{2}$ is calculated from Eq. (25) using the finite nucleus density $\rho(r)$ obtained from selfconsistent deformed Hartree-Fock plus BCS calculations with density-dependent Skyrme interactions. We use expressions for $S^{N M}(x)$ and $p_{0}^{N M}(x)$ derived using Bonn $\mathrm{B}$ and Bonn CD potentials in non-relativistic BruecknerHartee-Fock calculations [2] of ground-state properties of nuclear matter at different densities. The role of the CDFM to extract the NSE and pressure in finite nuclei from their counterparts in infinite nuclear matter is presented schematically by a block diagram in Fig. 4 .

\section{RESULTS OF CALCULATIONS}

In this Section we present the obtained results for the symmetry energy and pressure in finite nuclei extracted from nuclear matter many-body calculations using realistic Bonn B and Bonn CD potentials. We show also the results for the volume and surface contributions to the nuclear symmetry energy.

In Fig. 5 we plot the symmetry energy in nuclear matter as a function of density for both Bonn B and 


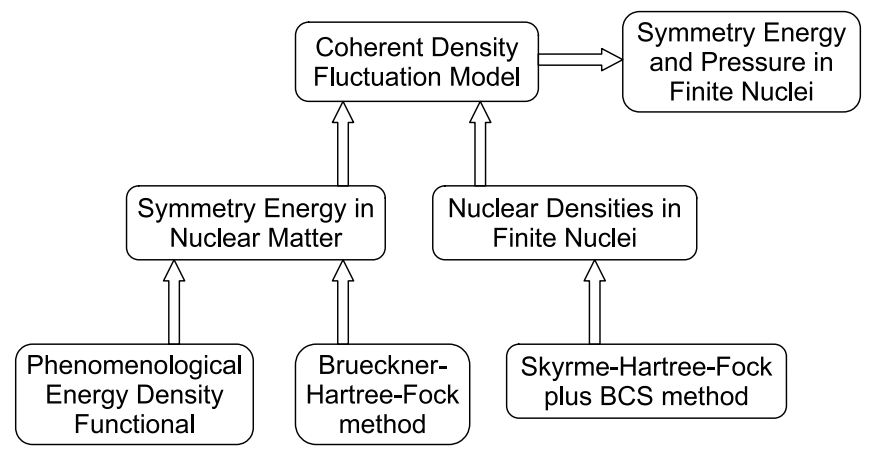

FIG. 4: Block diagram of the application of the CDFM for the extraction of symmetry energy and pressure in finite nuclei from their counterparts in nuclear matter.

Brueckner effective potential (from [16, 17]) in a range of nuclear densities where the expression of symmetry energy derived from the effective Brueckner potential is non-negative. On the same figure we present for a comparison two other density dependencies $S^{N M}(\rho)$ used in our work 37]. These are the power parametrization (see Refs. [45, 46, 49])

$$
S^{N M}(\rho)=S^{V}\left(\frac{\rho}{\rho_{0}}\right)^{\gamma}
$$

[noted in the figure by $S_{0}(\rho)$ ] for the case when $\gamma=0.40$ and also (Refs. [61, 62]):

$$
S^{N M}(\rho)=12.5\left(\frac{\rho}{\rho_{0}}\right)^{2 / 3}+17.6\left(\frac{\rho}{\rho_{0}}\right)^{\gamma}
$$

[noted in the figure by $\left.S_{4}(\rho)\right]$ for the case when $\gamma=0.30$. The data for the symmetry energy obtained in Ref. 63] from nuclear isobaric analog states (IAS) and from neutron skin thickness $\Delta r_{n p}$ of heavy nuclei $\left(\mathrm{IAS}+\Delta r_{n p}\right)$ [64, 65] are also presented in Fig. .5 by grey hatched and magenta bands, respectively. The two bands are taken from Ref. [66], where the role of the three-body forces have been studied. As can be seen from Fig. 5 , the symmetry energy calculated with Bonn B potential is slightly smaller for $\rho \leq 0.25 \mathrm{fm}^{-3}$ than the ones obtained from the Brueckner EDF and using the power parametrization [Eqs. (36) and (37)]. Nevertheless, the behavior of all symmetry energy curves is similar and in accordance with the empirical data [63 65] around the normal NM density.

In Fig. 6 we overlay the surface part of the density distribution of ${ }^{78} \mathrm{Ni}$ and the corresponding CDFM weight function $|\mathcal{F}(x)|^{2}$ as a function of $x$. The density is obtained in a self-consistent Hartree-Fock + BCS calculations with SLy4 interaction. The function $|\mathcal{F}(x)|^{2}$ which is used in Eqs. (27), (28), and (35) has the form of a bell with a maximum around $x=R_{1 / 2}$ at which the value of the density $\rho\left(x=R_{1 / 2}\right)$ is around half of the value of the central density equal to $\rho_{0}\left[\rho\left(R_{1 / 2}\right) / \rho_{0}=0.5\right]$. Namely in this region around $\rho=0.5 \rho_{0}$ the values of

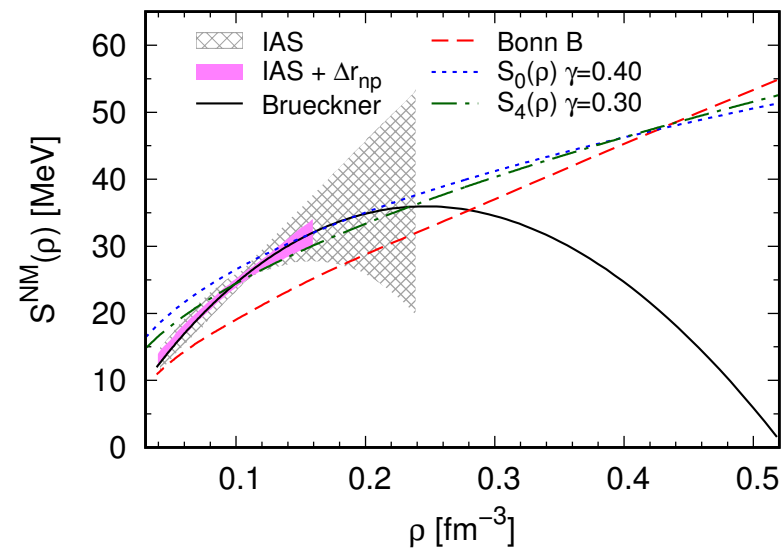

FIG. 5: Density dependence of the symmetry energy $S^{N M}(\rho)$ in nuclear matter with Brueckner and Bonn B potentials 16 , 17]. The curves for $S_{0}(\rho)(\gamma=0.40)$ [Eq. (36)] and $S_{4}(\rho)$ $(\gamma=0.30)[$ Eq. (37)] are also given. The constraints on the symmetry energy taken from Fig. 4 of Ref. [66] are presented by two bands (see the text).

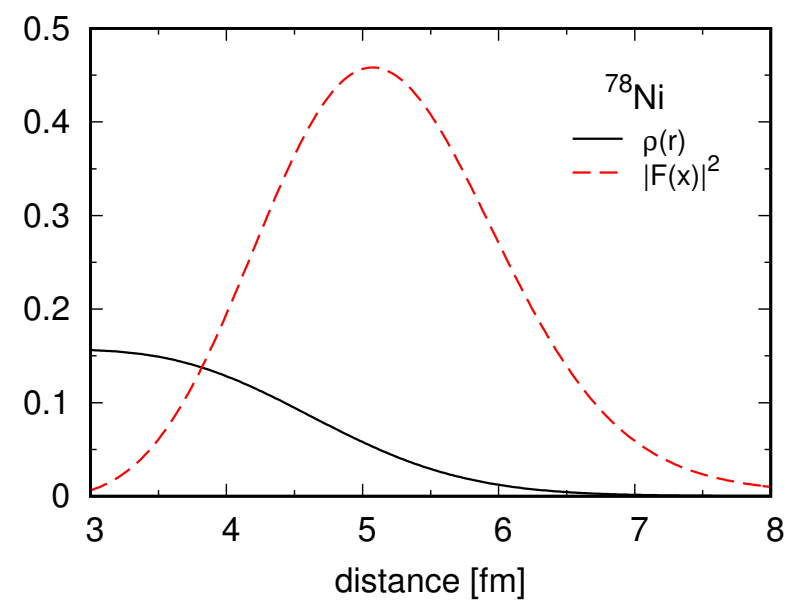

FIG. 6: The density $\rho(r)$ (in $\mathrm{fm}^{-3}$ ) of ${ }^{78} \mathrm{Ni}$ calculated in the Skyrme HF + BCS method with SLy4 force (normalized to $A=78)$ and the weight function $|\mathcal{F}(x)|^{2}\left(\right.$ in $\left.^{-1}\right)$ normalized to unity [Eq. (26)].

different $S^{N M}(\rho)$ play the main role in the calculations. As it is known, the central density of the nucleus has values around $\rho_{0} \approx 0.10-0.16 \mathrm{fm}^{-3}$. Consequently, the maximum of the weight function $|\mathcal{F}(x)|^{2}$ is around $\rho\left(R_{1 / 2}\right) \approx 0.05-0.08 \mathrm{fm}^{-3}$. In the case of ${ }^{78} \mathrm{Ni}$ (Fig. 6) the maximum of $|\mathcal{F}(x)|^{2}$ is at $\rho=0.05 \mathrm{fm}^{-3}$ and, within its width range, the density $\rho$ is between $0.12 \mathrm{fm}^{-3}$ and $0.01 \mathrm{fm}^{-3}$. Therefore, for the finite nucleus calculations the relevant values of $S^{N M}$ are typically those in the region around $\rho \approx 0.01-0.12 \mathrm{fm}^{-3}$ in Figs. 1 and 5 ,

We apply the CDFM to derive the values of the symmetry energy and pressure in three isotopic chains of nuclei $(\mathrm{Ni}, \mathrm{Sn}$, and $\mathrm{Pb})$ from our many-body symmetry energy calculations in nuclear matter with Bonn B and Bonn CD potentials from [16, 17] with input nuclear densities 
obtained from Hartree-Fock + BCS self-consistent calculations with different Skyrme paramatrizations. We use effective Skyrme forces SLy4 [67], Sk3 68], and SGII [69], namely because they are among the most widely used Skyrme forces and have been already used in our previous paper [70].

Using Eqs. (27) and (28) we calculate the nuclear symmetry energy and the pressure, correspondingly, as well as by means of Eqs. (35), (10), and (11) the volume and surface contributions to the symmetry energy in finite nuclei.

We next comment on the similarities and differences between our results for the symmetry energy (along with its volume and surface components) and pressure in the isotopes of $\mathrm{Ni}, \mathrm{Sn}$, and $\mathrm{Pb}$ nuclei. As can be seen in Fig.4 we use the CDFM that links the microscopic description of finite nuclei obtained from Skyrme-Hartree-Fock plus BCS method with the symmetry of nuclear matter. In this work the latter is obtained in two different ways, namely by self-consistent many-body Brueckner-HartreeFock calculations with realistic Bonn B and Bonn CD potentials or by effective Brueckner density functional. In this way the CDFM used in our hybrid approach utilizes the strong sides of both the ab initio many-body Brueckner-Hartree-Fock calculations using Bonn B and Bonn CD potentials (which give reasonable results for the binding energy in nuclear matter at saturation densities) and the Skyrme-Hartree-Fock + BCS method which gives realistic results for the nuclear density profiles in finite nuclei with appropriate choice of the parameters of the density-dependent Skyrme contact interaction (SLy4, Sk3, and SGII).

In the case of the $\mathrm{Ni}$ isotopic chain (Fig. 7) for the choice of the Brueckner EDF the calculated symmetry energy (using the CDFM with the SLy4 force) is in the range 26.8-27.8 MeV. The volume symmetry energy $S^{V}$ is within $41.7-42.3 \mathrm{MeV}$ and the surface symmetry energy $S^{S}$ in the range $17-19 \mathrm{MeV}$. The pressure is in the range $1.39-1.48 \mathrm{MeV} / \mathrm{fm}^{3}$ and the ratio $\kappa$ within $2.22-2.44$. By inspecting the graphs (Fig. 7) of the values of these nuclear state parameters in $\mathrm{Ni}$ isotopes one notices that at $A=78$ the symmetry energy $S(A)$, as well as its volume and surface components, $S^{V}$ and $S^{S}$ and the pressure $p_{0}$, reach maximum values while $\kappa$ has a minimum $(\kappa=2.22)$.

For the choice of the Bonn CD one-boson exchange potential [17] the calculated symmetry energy (using the CDFM with the SLy4 force) is in the range of 25.4-25.9 $\mathrm{MeV}$ with volume symmetry energy $S^{V}$ within 28.4-29.1 $\mathrm{MeV}$ and surface symmetry energy $S^{S}$ in the range 14.115.6 MeV. The pressure is in the range $3.1-3.7 \mathrm{MeV} / \mathrm{fm}^{3}$ and the ratio $\kappa$ within 1.8-2.0. As can be seen in Fig. 5 , at $A=78$ the symmetry energy $S(A)$, as well as its volume and surface components, $S^{V}$ and $S^{S}$ and pressure $p_{0}$, reach minimum values while $\kappa$ has a weakly expressed maximum $(\kappa=2.0)$.

In the case of the Bonn B one-boson exchange potential [16] the result for the symmetry energy (using the CDFM with the SLy4 force) is in the range of $24.4-24.8 \mathrm{MeV}$, with volume symmetry energy $S^{V}$ within 27.6 and 28.1 $\mathrm{MeV}$ and surface symmetry energy $S^{S}$ within 14.9 and 16.0 MeV. The pressure is in the range $3.1-3.6 \mathrm{MeV} / \mathrm{fm}^{3}$, while $\kappa$ is within 1.8-1.9. One can see in Fig. 7 that at $A=78$ the symmetry energy $S(A)$ as well as its volume and surface components, $S^{V}$ and $S^{S}$ and pressure, $p_{0}$ reach minimum values while $\kappa$ has a very weak maximum $(\kappa=1.9)$.

The smaller values of the symmetry energies of NM observed in Fig. 5 at the range of densities relevant in the integration in Eq. (27) lead to smaller values for the NSE in the case of Bonn B and Bonn CD potentials from [16, 17] in comparison with the Brueckner potential in the $\mathrm{Ni}$ chain. At the same time, the almost identical density dependence of $p_{0}^{N M}(\rho)$ of both realistic potentials (see Fig. (2) produce similar values of $p_{0}$ for the Ni isotopes being larger than the Brueckner ones.

Here we note that the observed peaks in the NSE and its volume and surface components at $A=78$ (see Fig. (7) take place for all choices of Skyrme interaction parametrizations (SLy4, Sk3, and SGII). They are more pronounced for the choice of the Brueckner energy density functional and they are somewhat smoothed out and less pronounced for Bonn B and Bonn CD oneboson-exchange potentials. We attribute this peak to the abrupt nuclear density change that is characteristic for double-magic nuclei, such as ${ }^{78} \mathrm{Ni}$. We note that these results concern all three Skyrme forces used (SLy4, Sk3, and SGII).

Similar peaks in the symmetry energy and its surface and volume components as functions of the mass number are predicted at the double-magic nucleus with $A=132$ in Sn (Fig. 8) (and additional peak at the semi-magic nucleus with mass number $A=140$ observed for the volume contribution to the NSE) and no pronounced peak at the double-magic nucleus with $A=208$ in Pb (Fig. 9) for all three choices of the parametrization of the Skyrme interaction. These peaks are more pronounced for the Brueckner density functional and much less pronounced for the employed Bonn B and Bonn CD potentials [16, 17]. In addition, the conclusions drawn already on the magnitude of the NSE $S$ and pressure $p_{0}$ for Ni isotopes using the three potentials and three Skyrme forces are valid also for the cases of $\mathrm{Sn}$ and $\mathrm{Pb}$ isotopes.

Here we would like to note that the observed maxima and minima of the considered quantities on Figs. 7 9 had been found and discussed in our previous works [26, 27, 29, 37] (so-called there "kinks"), studying the density dependence of the NSE for $\mathrm{Ni}, \mathrm{Sn}$, and $\mathrm{Pb}$ isotopes (see, e.g., details in Ref. 27]).They are related to the shell effects which are important at zero temperature. As mentioned above, the observed behavior of $S, S^{V}, S^{S}$, and $\kappa$ can be attributed to the profiles of the density distributions, particularly in the surface region. We mention that even the small differences between the densities of the double-magic nuclei ${ }^{78} \mathrm{Ni},{ }^{132} \mathrm{Sn}$ (and also of the semi-magic ${ }^{140} \mathrm{Sn}$ where the neutron shell $2 f_{7 / 2}$ is closed), as well as ${ }^{208} \mathrm{~Pb}$, and the neighbor nuclei lead through 

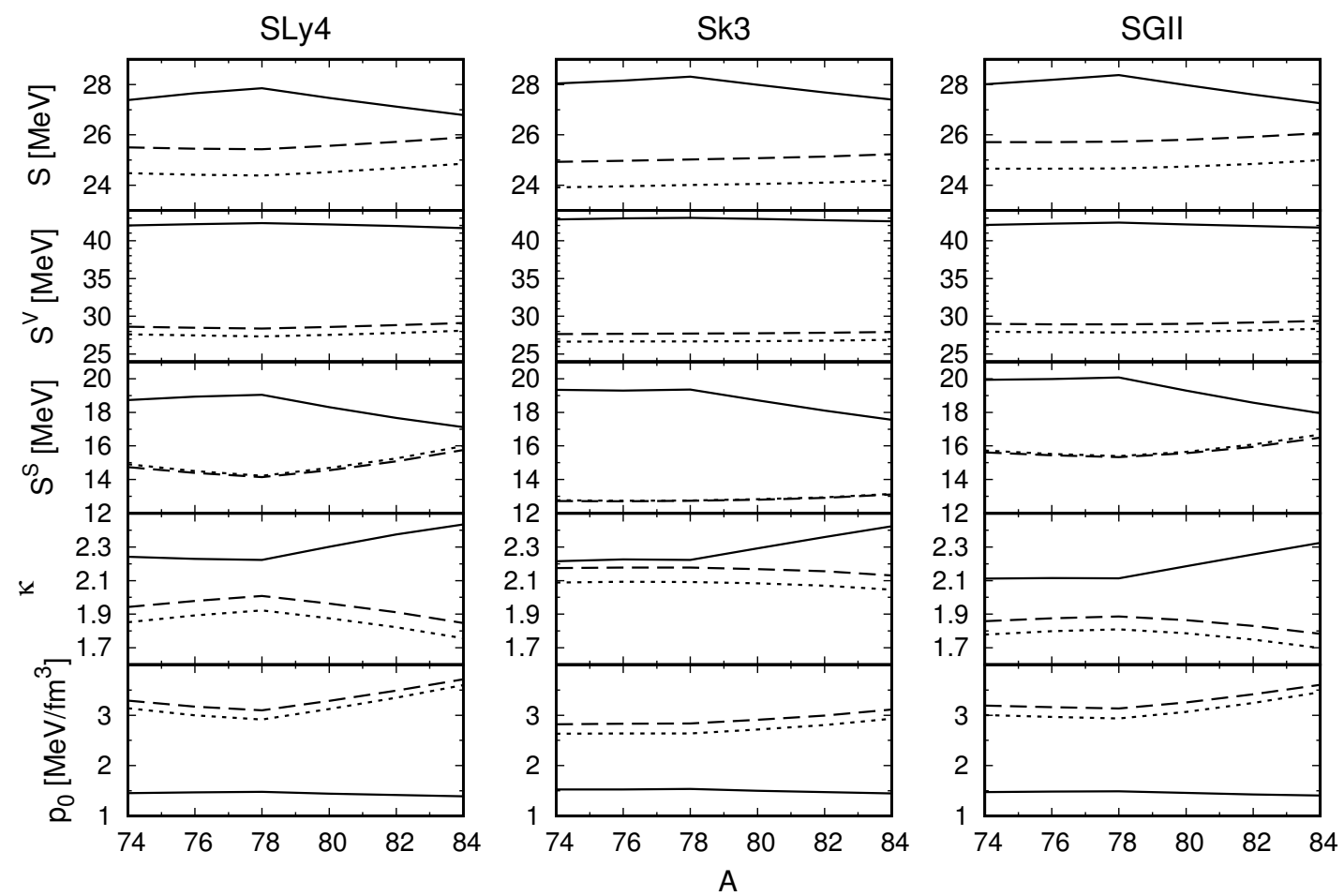

FIG. 7: The symmetry energy $S$, its volume $S^{V}$ and surface $S^{S}$ components, their ratio $\kappa$ and the pressure $p_{0}$ for Ni isotopes obtained using Brueckner EDF (solid line) and BHF method with Bonn CD (dashed line) and Bonn B (dotted line) potentials from [16, 17]. The weight function $|\mathcal{F}(x)|^{2}$ used in the calculations is obtained by means of the densities derived within a selfconsistent Skyrme-Hartree-Fock plus BCS method with SLy4 (left panel), Sk3 (middle panel) and SGII (right panel) Skyrme interactions.

their derivative to larger differences of the weight function $|\mathcal{F}(x)|^{2}$ [see Eq. (25)]. The latter is one of the main ingredients in the calculations of the quantities studied in our work [see Eqs. (27), (28), and (35)].

Here we would like to note that in some cases the observed peculiarities (maxima and minima, the so called kinks) in Figs. 7 and 8 are different, from one side, when the Bonn B and Bonn CD potentials are used and, from other side, in the case of the Brueckner EDF. These are, for example, the cases of $S^{S}, \kappa$ and $p_{0}$ (with the SLy4 force) for $\mathrm{Ni}$ isotopes and $S, S^{S}, \kappa$ and $p_{0}$ in Fig. 8 for Sn isotopes. Thus, within our method, in addition to the profiles of the density distributions, the reason for the mentioned differences is also related to the model used to calculate the nuclear matter properties that enter the calculations of the corresponding finite nuclear properties under study.

The values of the symmetry energy, of its volume and surface components and their ratio obtained in our work and shown above are, in general, compatible with those quoted in the existing literature (see, e.g., [4648, 63, 71]). The latter are, for example, the empirical value of the symmetry energy $30 \pm 4 \mathrm{MeV}$ given in Refs. [71, 72], the values of the volume symmetry energy (between 27 and $33.7 \mathrm{MeV}$ ) [46 48, 63] and the surface symmetry energy (between 9 and $12 \mathrm{MeV}$ ) [48]. In addition, the published values of $\kappa$ extracted from nu- clear properties, such as the isobaric analog states and skins [46] and masses and skins [47], being presented in [49], are $2.0 \leq \kappa \leq 2.8$. Another range of values of $\kappa$ $(1.6 \leq \kappa \leq 2.0)$ is also given in Ref. [49].

Considering in more details the comparison of our results for the NSE and its components with the existent data mentioned above we note that the main differences exist in the following cases: i) for the surface component of NSE $S^{S}$ between 14.1 and $14.6 \mathrm{MeV}$ when the Bonn $\mathrm{CD}$ potential is used and between 14.9 and 16.0 in the case of the Bonn B potential, and ii) for the NSE $S$ (between 24.4 and $24.8 \mathrm{MeV}$ ) when the Bonn B potential is used.

The values of the pressure obtained in our work for the three chains of isotopes $(\mathrm{Ni}, \mathrm{Sn}$, and $\mathrm{Pb})$ using Bonn $\mathrm{B}$ and Bonn CD potentials from [16, 17] are around 3 $\mathrm{MeV} / \mathrm{fm}^{3}$. This value is close to the upper limit of the range of $1.95-2.95 \mathrm{MeV} / \mathrm{fm}^{3}$ of theoretical estimates in other Brueckner-Hartree-Fock calculations [41] with various versions of Argonne $V_{18}$ potentials and is compatible with the value $p_{0}=2.3 \pm 0.8 \mathrm{MeV} / \mathrm{fm}^{3}$ extracted from measurements of the strength of the pygmy dipole resonance in $\mathrm{Sn}$ and $\mathrm{Pb}$ isotopes [73]. However, the value of $p_{0}$ obtained in our work when the Brueckner EDF is used is roughly twice less than those obtained when we use Bonn B and Bonn CD potentials 16, 17] and than the already mentioned values in Refs. [41] and 73. 

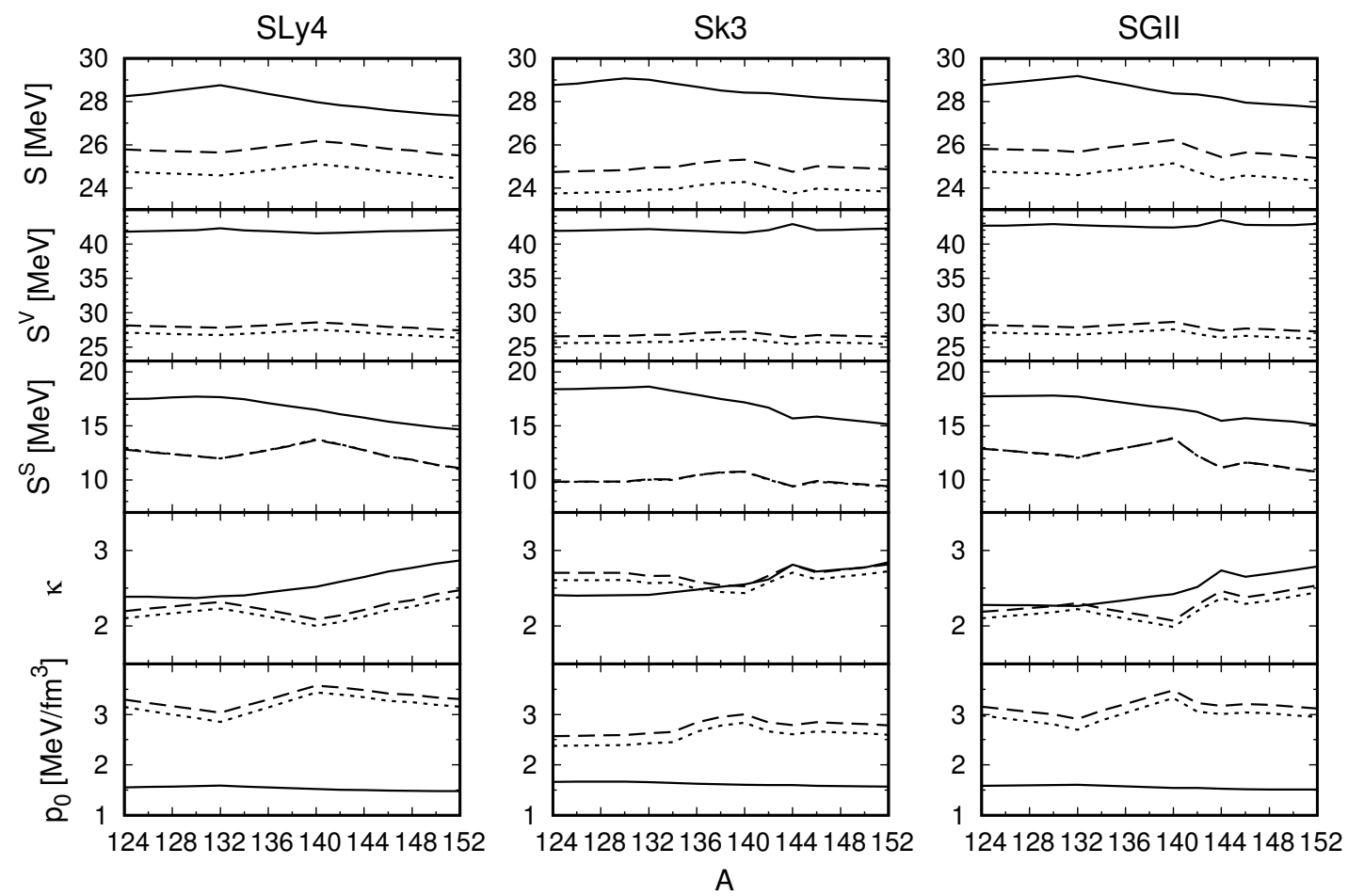

FIG. 8: Same as Fig. [7 but for Sn isotopes.

In addition, the values of the nuclear pressure $p_{0}$ obtained in our approach with the same potentials for ${ }^{208} \mathrm{~Pb}$ nucleus agree well with the recent results for the slope parameter $L$ between 44 and $58 \mathrm{MeV}$ [74] that correspond to $p_{0}$ between 2.35 and $3.09 \mathrm{MeV} / \mathrm{fm}^{3}$. The latter values obtained from most recent EOS based on state-of-theart chiral $N N$ potentials were extracted from the typical correlation between $L$ and the thickness of the neutron skin in ${ }^{208} \mathrm{~Pb}$ (between approximately 0.14 and $0.16 \mathrm{fm}$ ).

An extension to include TBF in finite systems, in particular in medium and heavy neutron-rich nuclei, for analysis of EOS properties of these nuclei in the framework of the BHF is an important task. In this case, the application of the CDFM could be justified a posteriori as a good choice to calculate the finite nuclei properties also when including three-body forces. Therefore, as a first step in this direction we give, as an example, estimations for the symmetry energy, its volume and surface contributions and their ratio, as well as the pressure, in the case of $\mathrm{Ni}$ isotopes on top of BHF calculations by including the corresponding self-consistent three-body forces. For this purpose we employ, as examples, the considerations from Ref. [54] where the BHF method with Bonn $\mathrm{B}$ potential is used, as well as from Ref. [55] where the in-medium T-matrix approach and Bonn CD potential is used. The role of the TBF in the case of the Bonn B potential [Fig. 10(a)] is to increase the symmetry energy $S$ for Ni isotopes with $A=74-84$ from $26.92-26.57$ $\mathrm{MeV}$ to $27.17-27.01 \mathrm{MeV}$, to change $S^{V}$ from 30.00 $29.52 \mathrm{MeV}$ to $29.93-29.70 \mathrm{MeV}$, to decrease $S^{S}$ from 14.33 - $14.32 \mathrm{MeV}$ to $12.74-12.95 \mathrm{MeV}$, as well as to in- crease $\kappa$ from $2.09-2.06$ to $2.34-2.29$ and $p_{0}$ from 3.34 - 3.30 to $5.08-5.42 \mathrm{MeV} / \mathrm{fm}^{3}$. We note the existence of the maxima of $S$ and $S^{V}$ for ${ }^{78} \mathrm{Ni}$ in both cases of twoand three- body forces and of $S^{S}$ in the case of two-body forces. The behaviour of the calculated characteristics for the same $\mathrm{Ni}$ isotopes when TBF are included can be seen in the case of the Bonn CD in Fig. 10(b). However, there is a bigger increase of $S$ from $27.62-27.19 \mathrm{MeV}$ to $38.02-$ $37.65 \mathrm{MeV}$, an increase of $S^{V}$ from $31.57-30.98 \mathrm{MeV}$ to 41.09-40.58 MeV, a decrease of $S^{S}$ from $18.98-18.93 \mathrm{MeV}$ to $13.92-13.86 \mathrm{MeV}$, an increase of $\kappa$ from $1.664-1.636$ to $2.95-2.93$, and strong one of $p_{0}$ from $2.86-2.911 \mathrm{MeV} / \mathrm{fm}^{3}$ to $6.31-6.49 \mathrm{MeV} / \mathrm{fm}^{3}$. One can see maxima for two- and three-body forces at $A=78$ in the cases of $S, S^{V}$, and $\kappa$ and a maximum of $S^{S}$ only in the case of TBF. The differences between the results obtained with the use of Bonn $\mathrm{B}$ and Bonn CD potentials plus TBF can be attributed to different EOS of nuclear matter (see Figs. 1 and 3) being stiffer for the Bonn CD potential. In addition, we have to note the role of the weight function $|\mathcal{F}(x)|^{2}$ when averaging the NM properties for both potentials plus TBF to obtain the considered quantities for the $\mathrm{Ni}$ isotopes in the CDFM scheme. Although the TBF affect considerably the high-density behavior of the symmetry energy of nuclear matter, their role on the symmetry energy and related quantities is not so large at subsaturation densities relevant for finite nuclei. This is due to the fact that the CDFM weight function $|\mathcal{F}(x)|^{2}$ in our approach has a bell form (see Fig. 6), which is peaked at distances that correspond to a density of around $\rho \approx 0.05-0.08$ $\mathrm{fm}^{-3}$ and its width is within the density range between 

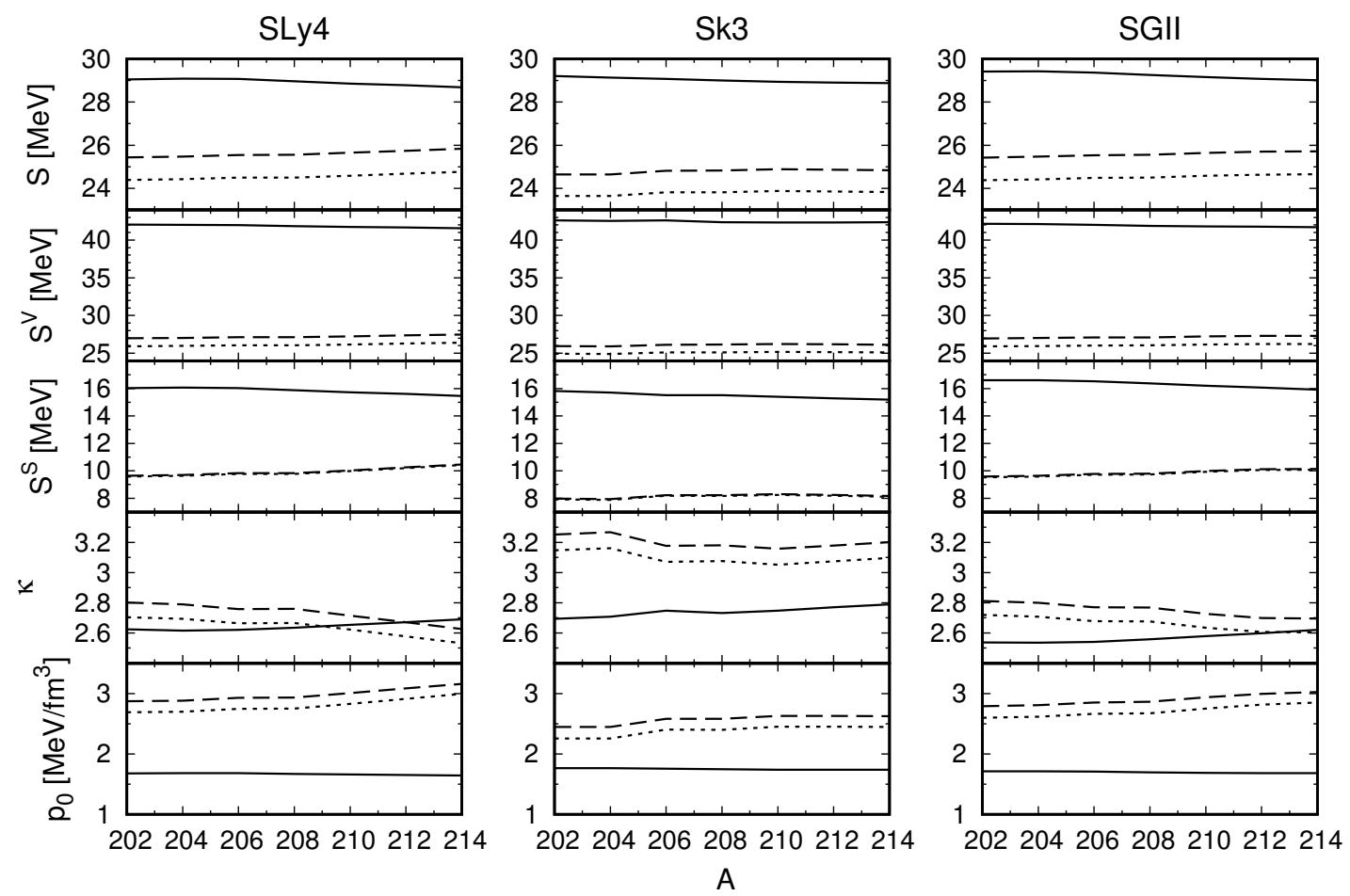

FIG. 9: Same as Fig. 7 but for $\mathrm{Pb}$ isotopes.

0.12 and $0.01 \mathrm{fm}^{-3}$. Namely in this region, where (as can be seen from Figs. 1 and 3) the difference between the values of $S^{N M}(\rho)$ in cases of both Bonn B and Bonn $\mathrm{CD}$ potentials when the two- and three-body forces are used is not so large. The values of the weight function are going to zero at densities $\rho>0.12 \mathrm{fm}^{-3}$ and $\rho<0.01$ $\mathrm{fm}^{-3}$ and that is why the differences between the values of $S^{N M}(\rho)$ in the cases of two- and three-body forces for nuclear matter at such densities are strongly reduced in finite nuclei.

\section{CONCLUSIONS}

In the present work we combine the non-relativistic Brueckner-Hartree-Fock method with the realistic Bonn $\mathrm{B}$ and Bonn CD $N N$ potentials with the CDFM within the self-consistent Skyrme-Hartree-Fock plus BCS approach to calculate the nuclear symmetry energy, its volume and surface components and their ratio, as well as the pressure for three isotopic chains of spherical nuclei $(\mathrm{Ni}, \mathrm{Sn}$, and $\mathrm{Pb})$. We find that the values of the NSE obtained in the BHF method are consistent and within 1.0-2.0 MeV agreement with the values obtained with the effective Brueckner potential. The calculated volume and surface components of the NSE for the $\mathrm{Ni}, \mathrm{Sn}$, and $\mathrm{Pb}$ chains obtained from the realistic Bonn B and Bonn CD two-body potentials are in a reasonable agreement with recently published estimations and available experimental data.

The results of our calculations of the pressure using
Bonn B and Bonn CD potentials [16, 17] turn out to be close to the upper limit of other BHF calculations using various versions of Argonne $V_{18}$ potentials and are comparable with the data from measurements of the strength of the pygmy dipole resonance in $\mathrm{Sn}$ and $\mathrm{Pb}$ isotopes. At the same time, our values of $p_{0}$ in the case when the Brueckner EDF is used are roughly twice less than those obtained when Bonn B and Bonn CD potentials are used and the values in Refs. [41, 73].

Complementary, in the present work we estimate within our approach the effects of three-body forces on the symmetry energy, its components and their ratio, as a first step on the example of $\mathrm{Ni}$ isotopes using the CDFM with BHF approximation with the Bonn B and Bonn CD potentials plus TBF [54, 55]. It is shown that the bell form of the CDFM weight function $|\mathcal{F}(x)|^{2}$ that is peaked around $\rho \approx 0.05-0.08 \mathrm{fm}^{-3}$ and whose values are going to zero for $\rho>0.12$ and $\rho<0.01 \mathrm{fm}^{-3}$, is the reason why the difference between the symmetry energy (and related quantities) for nuclear matter when the two- and threebody forces are used is much smaller in the case of finite nuclei. The small effect when TBF are included is better observed in the case of Bonn B three-body potential, where the values of the studied quantities are closer to the ones calculated by adopting purely the two-body forces. In our opinion, the role of microscopic three-body forces in the proposed approach to study the surface properties of neutron-rich nuclei can be clearly revealed in the future by applying, for instance, the latest version of the Barcelona-Catania-Paris-Madrid nuclear energy density functional (75] and references therein), which is con- 
(a)

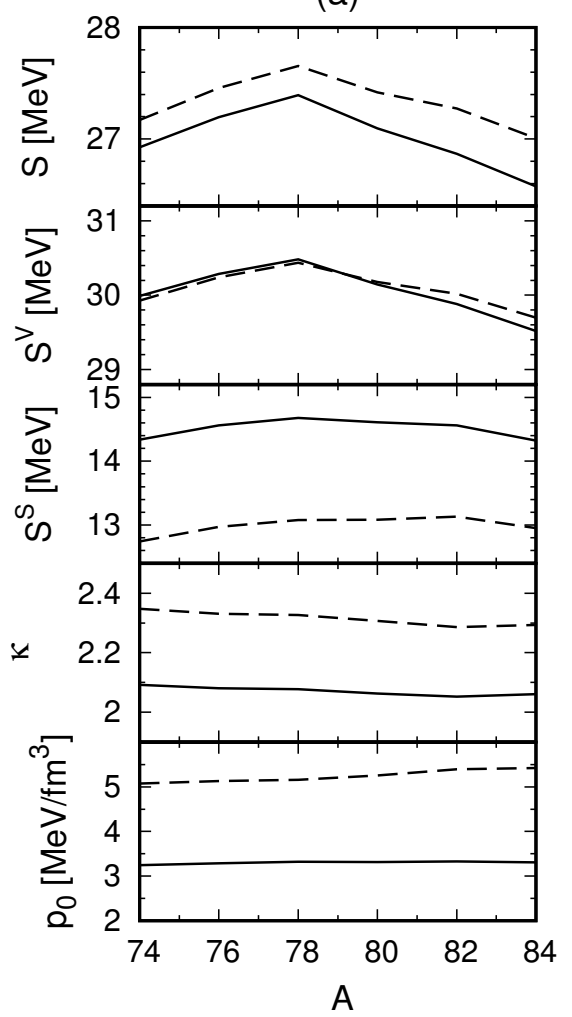

(b)

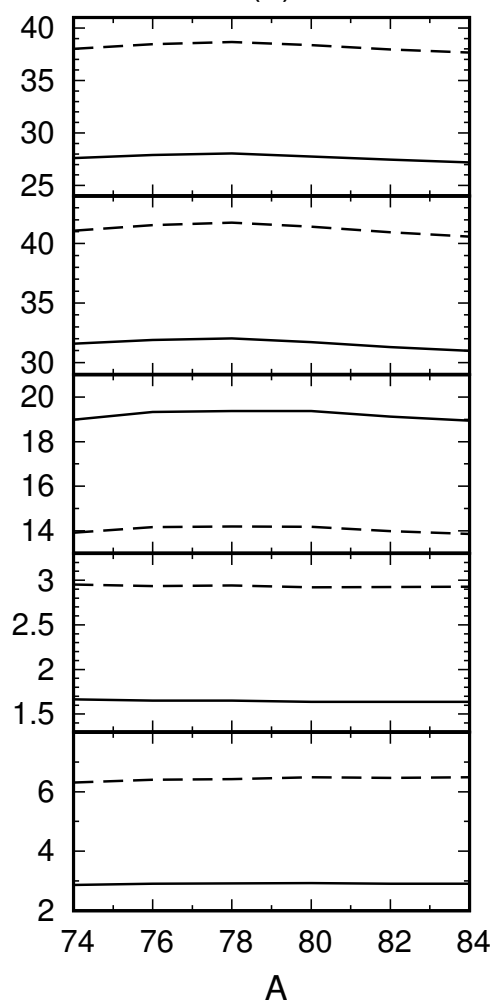

FIG. 10: The symmetry energy $S$, its volume $S^{V}$ and surface $S^{S}$ components, their ratio $\kappa$ and the pressure $p_{0}$ for Ni isotopes, obtained by means of the CDFM and different nuclear matter calculations using the Bonn B potential in the BHF method [54] (a) and the Bonn CD potential in the in-medium T-matrix method [55] (b) . Solid (dashed) line corresponds to the results in the case without (with) TBF contributions (see the text). The weight function $|\mathcal{F}(x)|^{2}$ used in the calculations is obtained by means of the densities derived within a self-consistent Skyrme-Hartree-Fock plus BCS method with SLy4 interaction.

structed upon the BHF calculations in nuclear matter and is able to treat successfully medium-heavy nuclei.

\section{Acknowledgments}

I.C.D would like to thank Professor Fred Myhrer and Professor Kuniharu Kubodera for numerous discussions and valuable advice and Professors Ruprecht Machleidt and Morten Hjorth-Jensen for their help in developing his computer code. I.C.D also wishes to acknowledge partial financial support from the University of Mount Olive Professional Development Fund. A.N.A, D.N.K., and M.K.G. are grateful for the support of the Bulgarian Science Fund under Contract No. KP-06-N38/1. P.S. acknowledges support from Ministerio de Ciencia, Innovación y Universidades MCIU/AEI/FEDER,UE (Spain) under Contract No. PGC2018-093636-B-I00.
[1] I. Bombaci and D. Logoteta, Astron. Astrophys. 609, A128 (2018).

[2] L. Engvik, M. Hjorth-Jensen, R. Machleidt, H. Müther, and A. Polls, Nucl. Phys. A 627, 85 (1997).

[3] R. B. Wiringa, V. Fiks, and A. Fabrocini, Phys. Rev. C 38, 1010 (1988).

[4] R. B. Wiringa, R. A. Smith, and T. L. Ainsworth, Phys. Rev. C 29, 1207 (1984).

[5] L. Engvik, E. Osnes, M. Hjorth-Jensen, G. Bao, and E. Østgaard, Ap. J. 469, 794 (1996).

[6] M. Baldo, I. Bombaci, and G. F. Burgio, Astron. Astrophys. 328, 274 (1997).
[7] R. V. Reid, Ann. Phys. 50, 411 (1968).

[8] M. Lacombe et al., Phys. Rev. C 21, 861 (1980).

[9] K. A. Brueckner, C. A. Levinson, Phys. Rev. 97, 1344 (1955).

[10] K. A. Brueckner, Phys. Rev. 97, 1353 (1955); Phys. Rev 100, 36 (1955).

[11] H. A. Bethe, Phys. Rev. 103, 1353 (1956).

[12] M. Haftel and F. Tabakin, Nucl. Phys. A 158, 1 (1970).

[13] K. Erkelenz, R. Alzetta, and K. Holinde, Nucl. Phys A 176, 413 (1971).

[14] K. Erkelenz, K. Holinde, and K. Bleuler, Nucl. Phys A 161, 155 (1971). 
[15] K. A. Brueckner, S. A. Coon, and J. Dabrowski, Phys. Rev. 168, 1184 (1968).

[16] R. Machleidt, Adv. Nucl. Phys. 19, 189 (1989).

[17] R. Machleidt, Phys. Rev. C 63, 024001 (2001).

[18] R. Machleidt, in Computational Nuclear Physics, edited by K. Langanke, J. A. Maruhn, and S. E. Koonin (Springer-Verlag, New York, 1993), Vol.2, p. 1.

[19] P. Prakash, Phys. Rep. 242, 191 (1994).

[20] C.-H. Lee, T. T. S. Kuo, G. Q. Li, and G. E. Brown, Phys. Rev. 57, 3488 (1998).

[21] P. Gögelein, E. N. E. van Dalen, Kh. Gad, Kh. S. A. Hassaneen, and H. Müther, Phys. Rev. C 79, 024308 (2009).

[22] Kh. S. A. Hassaneen, J. Mod. Phys. 5, 1713 (2014).

[23] Shihang Shen, Haozhao Liang, Wen Hui Long, Jie Meng, and Peter Ring, Prog. Part. Nucl. Phys. 109, 103713 (2019).

[24] A. N. Antonov, V. A. Nikolaev, and I. Zh. Petkov, Bulg. J. Phys. 6, 151 (1979); Z. Phys. A 297, 257 (1980); ibid 304, 239 (1982); Nuovo Cimento A 86, 23 (1985); A. N. Antonov et al., ibid 102, 1701 (1989); A. N. Antonov, D. N. Kadrev, and P. E. Hodgson, Phys. Rev. C 50, 164 (1994).

[25] A. N. Antonov, P. E. Hodgson, and I. Zh. Petkov, Nucleon Momentum and Density Distributions in Nuclei (Clarendon Press, Oxford, 1988); Nucleon Correlations in Nuclei (Springer-Verlag, Berlin-Heidelberg-New York, 1993).

[26] M. K. Gaidarov, A. N. Antonov, P. Sarriguren, and E. Moya de Guerra, Phys. Rev. C 84, 034316 (2011).

[27] M. K. Gaidarov, A. N. Antonov, P. Sarriguren, and E. Moya de Guerra, Phys. Rev. C 85, 064319 (2012).

[28] M. K. Gaidarov, P. Sarriguren, A. N. Antonov, and E. Moya de Guerra, Phys. Rev. C 89, 064301 (2014).

[29] A. N. Antonov, D. N. Kadrev, M. K. Gaidarov, P. Sarriguren, and E. Moya de Guerra, Phys. Rev. C 95, 024314 (2017).

[30] A. N. Antonov, M. K. Gaidarov, P. Sarriguren, and E. Moya de Guerra, Phys. Rev. C 94, 014319 (2016).

[31] K. A. Brueckner, J. R. Buchler, S. Jorna, and R. J. Lombard, Phys. Rev. 171, 1188 (1968).

[32] B. K. Agrawal, J. N. De, S. K. Samaddar, M. Centelles, and X. Viñas, Eur. Phys. J. A 50, 19 (2014).

[33] S. K. Samaddar, J. N. De, X. Viñas, and M. Centelles, Phys. Rev. C 76, 041602(R) (2007).

[34] S. K. Samaddar, J. N. De, X. Viñas, and M. Centelles, Phys. Rev. C 78, 034607 (2008).

[35] J. N. De and S. K. Samaddar, Phys. Rev. C 85, 024310 (2012).

[36] Ning Wang, Min Liu, Li Ou, and Yingxun Zhang, Phys. Lett. B 751, 553 (2015).

[37] A. N. Antonov, D. N. Kadrev, M. K. Gaidarov, P. Sarriguren, and E. Moya de Guerra, Phys. Rev. C 98, 054315 (2018).

[38] K. A. Brueckner, J. R. Buchler, R. C. Clark, and R. J. Lombard, Phys. Rev. 181, 1543 (1969).

[39] C. F. von Weizsäcker, Zeitschrift fuer Physik 96, 431 (1935).

[40] H. A. Bethe, Theory of Nuclear Matter, Annual Review of Nuclear Science, vol. 21, (Palo Alto, California, USA, 1971), p.93.

[41] A. E. L. Dieperink, Y. Dewulf, D. Van Neck, M. Waroquier, and V. Rodin, Phys. Rev. C 68, 064307 (2003).

[42] Lie-Wen Chen, Phys. Rev. C 83, 044308 (2011).
[43] W. D. Myers and W. J. Swiatecki, Ann. Phys. 55, 395 (1969).

[44] A. W. Steiner, M. Prakash, J. M. Lattimer, and P. J. Ellis, Phys. Rep. 411, 325 (2005).

[45] P. Danielewicz, arXiv: 0607030 [nucl-th].

[46] P. Danielewicz, arXiv: 0411115 [nucl-th].

[47] P. Danielewicz, Nucl. Phys. A 727, 233 (2003).

[48] P. Danielewicz and J. Lee, Nucl. Phys. A 818, 36 (2009).

[49] A. E. L. Dieperink and P. Van Isacker, Eur. Phys. J. A 32, 11 (2007).

[50] H. Q. Song and T. T. S. Kuo, Phys.Rev C 43, 2883 (1991).

[51] H. Q. Song, Z. X. Wang, and T. T. S. Kuo, Phys.Rev C 46, 1788 (1992).

[52] M.Baldo, in Nuclear Methods and the Nuclear Equation of State, ed. M.Baldo (River Edge, NJ, World Scientific, 1999), p.1

[53] M. Hjorth-Jensen, Lectures at the Twelfth Summer School in Nuclear Physics, University of California, Santa Cruz, July 3-July 14, 2000

[54] P. Wang and W. Zuo, Chin. Phys. C 38, 084102 (2014).

[55] V. Somà and P. Bożek, Phys. Rev. C 78, 054003 (2008).

[56] A. Akmal and V. R. Pandharipande, Phys. Rev. C 56, 2261 (1997).

[57] F. Coester, S. Cohen, B. Day, and C. M. Vincent, Phys. Rev. C 1, 769 (1970).

[58] M. Baldo and Alaa Eldeen Shaban, Phys. Lett. B 661, 373 (2008).

[59] S. Gandolfi, J. Carlson, and S. Reddy, Phys. Rev. C 85, 032801(R) (2012).

[60] J.J. Griffin and J.A. Wheeler, Phys. Rev. 108, 311 (1957).

[61] M. B. Tsang, Yingxun Zhang, P. Danielewicz, M. Famiano, Zhuxia Li, W. G. Lynch, and A. W. Steiner, Phys. Rev. Lett. 102, 122701 (2009); M. B. Tsang et al., Int. J. Mod. Phys. E 19, 1631 (2010).

[62] J. Dong, W. Zuo, J. Gu, and U. Lombardo, Phys. Rev. C 85, 034308 (2012).

[63] P. Danielewicz and J. Lee, Nucl. Phys. A 922, 1 (2014) (and references therein).

[64] X. Roca-Maza, M. Brenna, B. K. Agrawal, P. F. Bortignon, G. Colo, L. G. Cao, N. Paar, and D. Vretenar, Phys. Rev. C 87, 034301 (2013).

[65] Z. Zhang and L.-W. Chen, Phys. Lett. B 726, 234 (2013).

[66] D. Logoteta, Phys. Rev. C 100, 045803 (2019).

[67] E. Chabanat, P. Bonche, P. Haensel, J. Meyer, and R. Schaeffer, Nucl. Phys. A 635, 231 (1998).

[68] M. Beiner, H. Flocard, N. Van Giai, and P. Quentin, Nucl. Phys. A 238, 29 (1975).

[69] N. Van Giai and H. Sagawa, Phys. Lett. B 106, 379 (1981).

[70] P. Sarriguren, M. K. Gaidarov, E. Moya de Guerra, and A. N. Antonov, Phys. Rev. C 76, 044322 (2007).

[71] P. E. Haustein, At. Data Nucl. Data Tables 39, 185 (1988).

[72] N. Nikolov, N. Schunk, W. Nazarewich, M. Bender, and J. Pei, Phys. Rev. C 83, 034305 (2011).

[73] A. Klimkiewicz et al., Phys. Rev. C 76, 051603(R) (2007).

[74] F. Sammarruca and R. Millerson, Front. Phys. 7:213 (2019)

[75] B. K. Sharma, M. Centelles, X. Viñas, M. Baldo, and G. F. Burgio, Astron. Astrophys. 584, A103 (2015). 